\title{
Two Decades of Contemporary Surgery of Primary Cardiac Tumors
}

\author{
Khalil Jawad, $\mathrm{MD}^{1}$ Tamer Owais, $\mathrm{MD}^{2}$ Stephan Feder, $\mathrm{MD}^{1}$ Sven Lehmann, MD, $\mathrm{PhD}^{1}$ \\ Martin Misfeld, MD, PhD ${ }^{1}$ Jens Garbade, MD, $\mathrm{PhD}^{1}$ Michael Borger, MD, $\mathrm{PhD}^{1}$ \\ ${ }^{1}$ Department of Cardiac Surgery, Heart Center, University of Leipzig, \\ Leipzig, Germany \\ 2 Department of Cardiac Surgery, Central Hospital Bad Berka, Bad \\ Address for correspondence Khalil Jawad, MD, Department of \\ Cardiac Surgery, Heart Center, Leipzig University, Struempellstrasse \\ 39, 04289 Leipzig, Germany \\ Berka, Germany \\ (e-mail: khalil.jawad@medizin.uni-leipzig.de).
}

Surg J 2018;4:e176-e181.

\begin{abstract}
Keywords

- cardiac tumor

- minimally invasive heart surgery

- surgical outcome

Objective The decision to operate cardiac tumors is an issue of balancing surgical outcome and survival with quality of life (QOL). We report our single-center experience in managing primary cardiac tumors between 1994 and 2014.

Methods and Results In this study, 269 patients were subjected to our standardized operative protocols, preoperative preparations, postoperative follow-up, and consents of participation. Demographic and preoperative/intraoperative/postoperative variables were collected with focus on long-term follow-up and survival. A total of 72,000 cardiac procedures were performed within 20 years at our institution. Two hundred sixty-nine patients were diagnosed with primary cardiac tumors $(0.37 \%)$, with a male:female ratio of $1: 1.68$, mean age of $57.4 \pm 19.5$ years, and body mass index of $25.49 \pm 6.5$. The most presenting symptoms were dyspnea $(n=94)$, arrhythmias $(n=53)$, embolic event $(n=36)$, and chest pain $(n=29)$, and 33 patients were accidentally discovered. Isolated tumor excision and concomitant ablation were performed on 181 patients, while the rest needed additional procedures such as coronary artery bypass grafting $(n=27)$ or valve surgery $(n=61)$. Focus on pathology, tumor location was done reporting the commonest pathology such as myxoma $(n=177)$ and fibroelastoma $(n=56)$. The frequent site was the left atrium $(n=162)$. Our primary results showed incidence of bleeding in 9 patients (3.3\%), arrhythmias in 76 patients $(28.25 \%)$, and mortality in 49 patients $(18.2 \%)$. Five patients $(1.8 \%)$ showed recurrence and 220 patients (81.8\%) showed complaint-free survival.

Conclusion Complete excision of primary cardiac tumors is the golden rule in management as it improves survival and decreases morbidity expected from the progressing tumors process. The progression of minimally invasive techniques improves QOL and should be performed whenever possible.
\end{abstract}

Primary cardiac tumors can be classified as either benign/ malignant or primary/secondary of malignant pathology. Data from 22 large autopsy series reported by McAllister et al showed the frequency of primary cardiac tumors is $\sim 0.02 \%$, corresponding to 200 tumors in 1 million autopsies. ${ }^{1}$

received

March 31, 2018 accepted after revision August 20, 2018
DOI https://doi.org/

10.1055/s-0038-1673333. ISSN 2378-5128.
Around $75 \%$ are benign; nearly half are myxomas; and a majority of the rest are lipomas, papillary fibroelastomas, and rhabdomyomas. ${ }^{1}$ Sixty years ago in 1954, Crafoord $^{3}$ was the first to excise an atrial myxoma on cardiopulmonary bypass. $^{2}$ Today, cardiac tumors represent only $0.3 \%$ of all
Copyright $\odot 2018$ by Thieme Medical Publishers, Inc., 333 Seventh Avenue, New York, NY 10001, USA. Tel: +1(212) 584-4662.
License terms

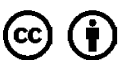


open-heart surgeries. ${ }^{3}$ The advent of modern surgical techniques, such as minimally invasive approaches, transformed the concept of high-risk surgery in cardiac tumor morbid patients into a surgery of lower and accepted risk. However, surgery is always indicated especially if obstructing intracardial flow or causing valvular dysfunction or conduction errors. ${ }^{4}$ This squeal denotes probably advanced tumorous stage, thereby limiting therapeutic options and expressing the benefits of minimally invasive approaches. ${ }^{5}$ In this report, we review the clinical and surgical experience and results of cardiac tumors at our Department of Cardiac Surgery, University of Leipzig, over the past 20 years. Survivors were contacted via telephone. The mean follow-up for this series is 5.3 years (range: $0.003-19.98$ years). Our focus in this study was relating the pathology, localization, symptoms, as well as age and gender distribution to the long-term results regarding survival, tumor relapse, and risk of recurrence matched to the corresponding neoplastic etiology using descriptive statistical measurements.

\section{Methods}

\section{Data Collection}

The medical records of the Leipzig Heart Center and the patient database of the Department of Cardiac Surgery were pro- and retrospectively reviewed to collect patients with primary cardiac tumors between 1994 and 2014. Approval to conduct this study was obtained by the Institutional Review Board of the University of Leipzig and patient consent was waived. All necessary detailed data concerning presentation, diagnosis, treatment, and follow-up were obtained. All patients with thrombus and tumors metastatic to the heart were excluded.

All survivors were contacted via telephone. For additional missing medical data, the family doctor in charge was contacted as well. Follow-up was performed for every patient. Survival data were completed with the nationwide statistical database, in which every death is recorded. The follow-up time was calculated either to death or to the last verified contact with the living patient. Median follow-up was $4.93 \pm 3.95$ years.

Treatment entailed complete medical-surgical protocols, beginning from only sternotomy or minimally invasive heart surgery tumor excision and/or adjuvant therapy for malignant tumors. After interdisciplinary discussion with the oncology department, adjuvant therapy for sarcoma patients was decided to be either a combination chemotherapy (six courses of ifosfamide $\left[1,500 \mathrm{mg} / \mathrm{m}^{2}\right.$, days $\left.1-4\right]$, dacarbazine $\left[200 \mathrm{mg} / \mathrm{m}^{2}\right.$, days $\left.1-4\right]$, and doxorubicin [25 $\mathrm{mg} / \mathrm{m}^{2}$, days $1-2$ ] administered in 14-day intervals and granulocyte colony-stimulating factor $\left[30 \times 10^{6} \mathrm{IU} / \mathrm{d}\right.$, subcutaneous] on days 5-13), or six courses of monochemotherapy (doxorubicin) and radiation, or six courses of monochemotherapy alone (doxorubicin or Herceptin), or radiation alone. Radiation dose was based on age, gender, and seize adjusted and ranged from a total dose of 15 to 60 Gy. Adjuvant therapy for our patients suffering from a nonHodgkin's lymphoma (diffuse large B cell lymphoma) con- sisted of six courses of rituximab, cyclophosphamide, doxorubicin, vincristine, and prednisone. Of the 269 patients, 100 were males and 169 were females. The youngest patient was 7 days and the oldest was 87 years (mean age: 57.4). Age-gender correlation, when taking all tumor pathologies into account, denoted that women were statistically significantly older $(p=0.02)$ than their male counterparts at the time of clinical presentation $(61.6 \pm 14.5$ vs. $51.6 \pm 18.7$ years $)$

Tumor characteristics are summarized in - Fig. 1. Among our study group, $65.8 \%$ were diagnosed as myxomas and $20.8 \%$ as fibroelastomas. The commonest location was the left atrium among 162 patients (60.2\%) and was mostly myxomas. The localization of fibroelastomas was more heterogeneous with a high affinity for aortic and mitral valve leaflets. Malignant cardiac tumors, whether sarcomas or lymphomas, composed a minor portion of our study population (14 patients [5.2\%]). The complete demographic profile of all patients is presented in -Table 1 .

\section{Preoperative Evaluation}

Related symptoms were dyspnea in 94 patients (34.9\%), general fatigue in 50 patients (18.6\%), central or peripheral neurological thromboembolic events in 36 patients (13.4\%), and angina in 29 patients (10.8\%). Subset of patients was asymptomatic and was accidentally discovered in 33 patients (12.3\%), whereas another subset was presenting with symptoms of congestive heart failure (20.1\%), who were in New York Heart Association (NYHA) class III or IV.

The presence of a tumor was diagnosed by echocardiogram. For morphological description in suspected cases, whether computed tomography or magnetic resonance imaging was performed. Coronary angiography was performed in all patients older than 40 years without risk factors (family history, dyslipidemia, and smoking) and in all patients older than 35 years in the presence of risk factors (-Table 2 ).

\section{Operative Techniques and Evaluation}

Principally, patients with cardiac tumors were subjected to surgery because of the possibility of embolic complications

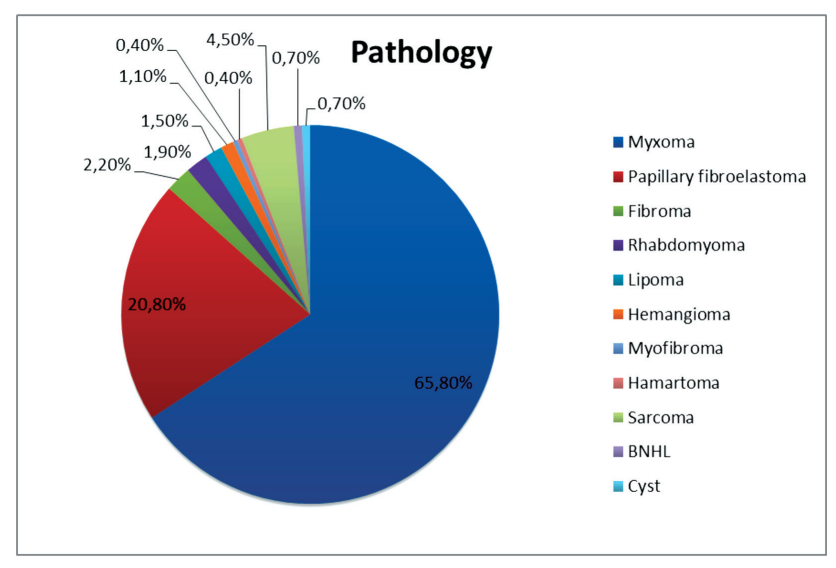

Fig. 1 Distribution of cardiac tumors. 
e178 Two Decades of Contemporary Surgery of Primary Cardiac Tumors Jawad et al.

Table 1 Baseline patient's characteristics

\begin{tabular}{|c|c|c|}
\hline Variables & $n(\%)$ & Mean (SD) \\
\hline Male & $100(37.2)$ & \\
\hline Female & $169(62.8)$ & \\
\hline \multicolumn{3}{|l|}{ Age $(y)$} \\
\hline$<20$ & $21(7.8)$ & \\
\hline $20-40$ & $16(5.9)$ & \\
\hline $40-60$ & $84(31.2)$ & \\
\hline $60-80$ & $142(52.7)$ & \\
\hline$>80$ & $6(2.2)$ & \\
\hline BMI $\left(\mathrm{kg} / \mathrm{m}^{2}\right)$ & & $25.49 \pm 6.5$ \\
\hline Hypertension & $161(60.1)$ & \\
\hline Diabetes & $53(19.8)$ & \\
\hline \multicolumn{3}{|c|}{ Left ventricular function } \\
\hline$<30 \%$ & $11(4.19)$ & \\
\hline $30-60 \%$ & $119(54.41)$ & \\
\hline$>60 \%$ & $132(49.07)$ & \\
\hline \multicolumn{3}{|c|}{ Presenting symptoms } \\
\hline Dyspnea & $34.9 \%$ & \\
\hline Chest pain & $10.8 \%$ & \\
\hline Syncope & $10 \%$ & \\
\hline Emboli & $13.4 \%$ & \\
\hline Arrhythmia & $21.2 \%$ & \\
\hline Accidentally & $12.3 \%$ & \\
\hline Vegetative & $18.6 \%$ & \\
\hline Others & $3.7 \%$ & \\
\hline
\end{tabular}

Abbreviations: BMI, body mass index; SD, standard deviation.

or sudden death. According to the morphological picture of the tumor and its localization, we decided whether to perform a midline sternotomy or a minimally invasive approach (either upper ministernotomy or right-sided lateral thoracotomy). Conduction of cardiopulmonary bypass was through the routine cannulation sites and under moderate systemic hypothermia, deep topical hypothermia, and cardioplegic cardiac arrest. The average bypass time was $94.01 \pm 206.2$ minutes and ischemic time was $42.57 \pm 30.8$ minutes. Incisions were mostly performed through the left atriotomy (57.99\%) and the second most common was the right atriotomy (28.99\%). Rare incisions were aortotomy $(9.29 \%)$, ventriculotomy $(1.85 \%)$, and pulmonary arteriotomy $(0.74 \%)$ according to the tumor localization. Detailed localization in the left atrium was not documented. Wide tumor excision with wide safety margins was our policy. In addition, cryoablation of the tumor base was done in cases where the tumor was showing deep intramyocardial invasion. Associated procedures included: coronary artery bypass grafting in 27 patients, mitral valve repair in 26 patients, mitral valve replacement in 6 patients, aortic valve replacement in 11 patients, aortic valve repair in
Table 2 Diagnostic

\begin{tabular}{|l|l|}
\hline & $\boldsymbol{n}(\%)$ \\
\hline $\mathrm{CT}$ & $3(1.1)$ \\
\hline Echo & $139(51.7)$ \\
\hline Echo/Angio & $30(11.2)$ \\
\hline Echo/Angio/CT & $7(2.6)$ \\
\hline Echo/CT & $28(10.4)$ \\
\hline Echo/CT/MRI & $16(5.9)$ \\
\hline Echo/MRI & $40(14.9)$ \\
\hline Echo/MRI/Angio & $4(1.5)$ \\
\hline Echo/PET/CT & $1(0.4)$ \\
\hline MRI & $1(0.4)$ \\
\hline All & $269(100)$ \\
\hline
\end{tabular}

Abbreviations: Angio, angiography; CT, computed tomography; Echo, echocardiography; MRI, magnetic resonance imaging; PET, positron emission tomography.

2 patients, tricuspid valve replacement in 1 patient, and

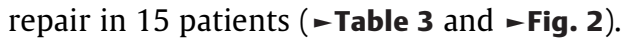

\section{Statistical Analysis}

Standard definitions were used for patient variables and outcomes. Categorical variables were expressed as percentages and continuous variables as mean \pm standard deviation

Table 3 Operating data

\begin{tabular}{|l|l|l|}
\hline & $\boldsymbol{n}(\%)$ & Mean (SD) \\
\hline Cross-clamp time (min) & & $42.57 \pm 30.83$ \\
\hline CPB time (min) & & $94.01 \pm 206.29$ \\
\hline Concomitant procedure & \multicolumn{2}{|l|}{} \\
\hline CABG & $27(10)$ & \\
\hline AV replacement & $11(4.1)$ & \\
\hline AV repair & $2(0.7)$ & \\
\hline MV replacement & $6(2.2)$ & \\
\hline MV repair & $26(9.7)$ & \\
\hline TV replacement & $1(0.4)$ & \\
\hline TV repair & $15(5.6)$ & \\
\hline Morrow resection & $4(1.5)$ & \\
\hline Tumor location & & \\
\hline Left atrium & $162(60.2)$ & \\
\hline Left ventricular & $26(9.7)$ & \\
\hline Valves & $37(13.7)$ & \\
\hline Right atrium & $33(12.3)$ & \\
\hline Right ventricular & $6(2.2)$ & \\
\hline Others & $5(1.9)$ & \\
\hline
\end{tabular}

Abbreviations: $\mathrm{AV}$, aortic valve; $\mathrm{CABG}$, coronary artery bypass grafting; $\mathrm{CPB}$, cardiopulmonary bypass; MV, mitral valve; TV, tricuspid valve. 


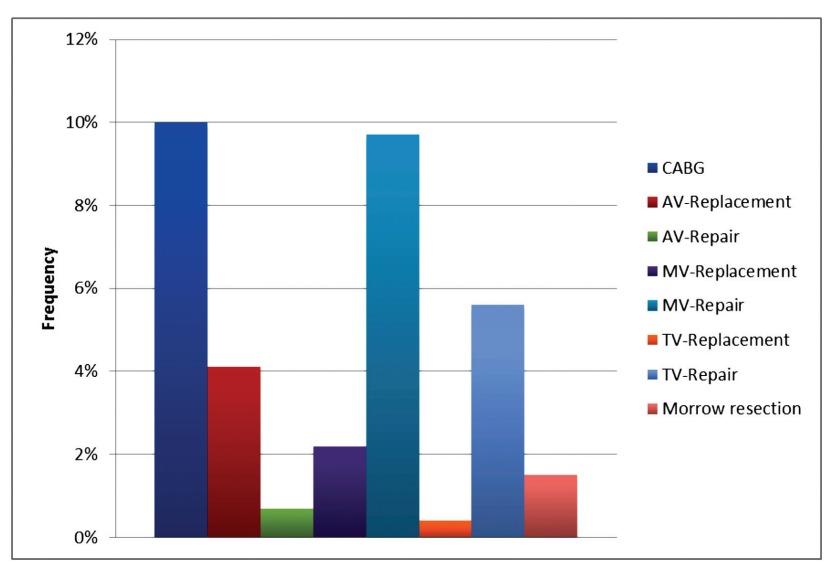

Fig. 2 Concomitant procedure.AV, aortic valve; $C A B G$, coronary artery bypass grafting; MV, mitral valve; TV, tricuspid valve.

(range). All statistical analyses were performed using IBM SPSS version 19.0 software (IBM Corp., New York). Comparisons of the preoperative and follow-up results were performed using a two-paired $t$-test and the Wilcoxon's signed rank test, respectively. Long-term survival of groups was evaluated and compared using the Kaplan-Meier's survival plot and the log-rank test, respectively. A two-sided $p$-value $<0.05$ was considered to be statistically significant.

\section{Results}

From 1994 to 2014, 72,000 cardiac procedures were performed at our institution. Among these procedures, a total of 269 patients were operated due to cardiac tumor masses (incidence $0.37 \%$ ); $73 \%$ of the patients were women. Among our study group, $65.8 \%$ were diagnosed as myxomas and $20.8 \%$ as fibroelastomas. The commonest location was the left atrium among 162 patients (60.2\%) and was mostly myxomas. The localization of fibroelastomas was more heterogeneous with a high affinity for aortic and mitral valve leaflets. According to the morphological picture of the tumor and its localization, we decided whether to perform a midline sternotomy (43.1\%) or a minimally invasive approach (56.8\%, either upper ministernotomy or right-sided lateral thoracotomy). Early postoperative results showed atrial or ventricular arrhythmias in 76 patients (28.3\%), syncope in 27 patients $(10.03 \%)$, respiratory insufficiency in 20 patients (7.4\%), occurrence of bleeding in 9 patients (3.3\%), renal insufficiency in 9 patients (3.3\%), pericardial effusion also in 9 patients (3.3\%), and mesenteric vascular occlusion in 3 patients $(1.1 \%)$ (-Fig. 3). No intraoperative mortality was recorded. In-hospital mortality was 5 patients $(1.9 \%)$ and mortality during follow-up was 49 patients $(18.2 \%)$ with follow-up period ranged from 0.003 to 19.98 years (-Fig. 4). In our study, we correlated the incidence of survival with various parameters such as age, gender, pathology, preoperative NYHA class and ejection fraction, postoperative bleeding and arrhythmias, and finally tumor localization. Our follow-up and statistical analysis showed that age, pathology, and NYHA class are dependent factors affecting survival (-Table 4).

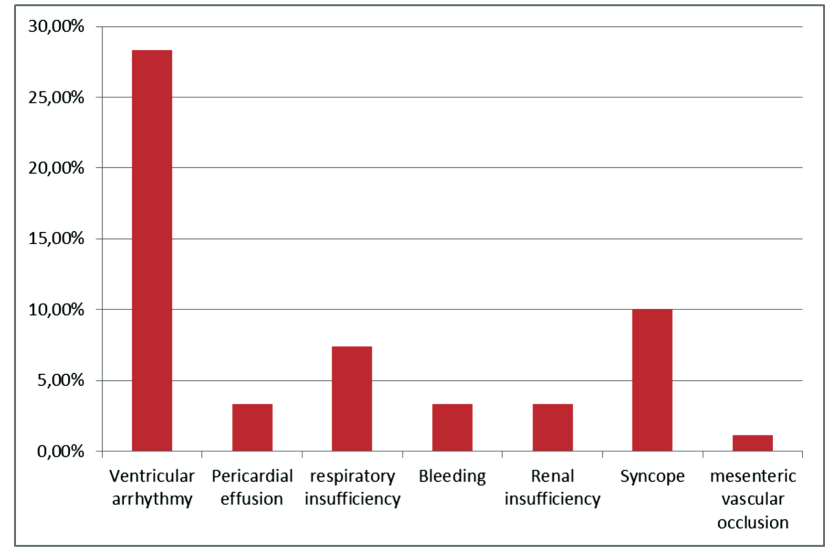

Fig. 3 Postoperative complications.

\section{Discussion}

In our single-center long-term survey of patients with cardiac masses, we have shown a very low incidence of this disease with excellent surgical results and low morbidity and mortality.

Our age-gender correlation reported a statistically significant older age in females to males. This coincides with the findings of the University of Vienna in 2015. ${ }^{6}$

It is worth noting that we did not exclude any patient from our study group based on gender. Our excluded patients were those with secondary metastasis and two patients with

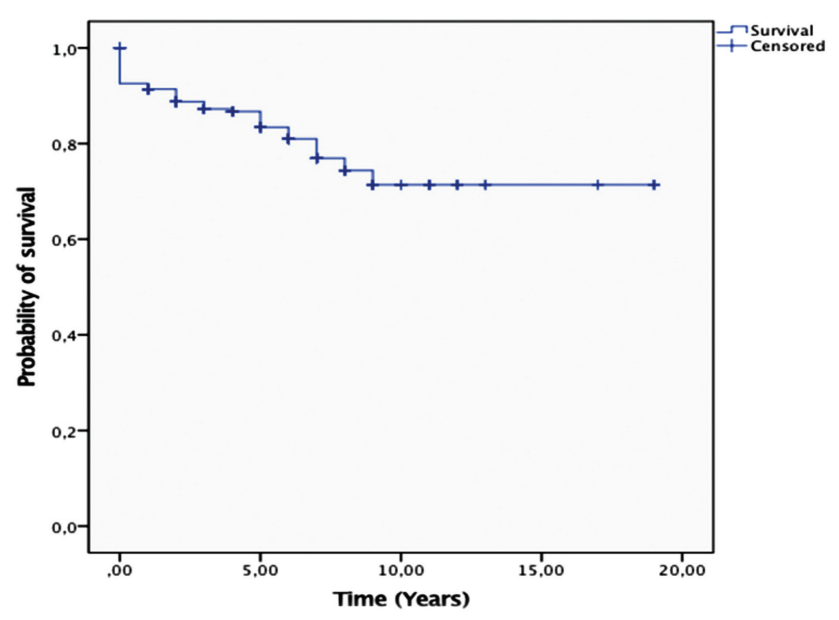

Fig. 4 Kaplan-Meier's survival analysis: all tumors.

Table 4 Cox regression analysis

\begin{tabular}{|l|l|l|}
\hline Variable & HR $(95 \% \mathrm{Cl})$ & $p$-Value \\
\hline Age & $2.320(1.14-4.68)$ & 0.019 \\
\hline Pathology & $1.238(1.15-1.33)$ & $<0.001$ \\
\hline NYHA & $1.392(1.02-1.9)$ & 0.037 \\
\hline
\end{tabular}

Abbreviations: $\mathrm{Cl}$, confidence interval; $\mathrm{HR}$, hazard ratio; NYHA, New York Heart Association. 
primary cardiac tumors, one of them was a male with heart failure and mechanically ventilated on high doses of vasopressors and the other was a female with a EuroSCORE of 39 and mechanically resuscitated on admission with persistent metabolic acidosis and nearly in a terminal clinical state.

Throughout our study group, we found that the commonest symptom was dyspnea (34.9\%), followed by constitutional manifestations (18.6\%) and the third commonest was accidental discovery of the tumor as well as emboli (13.4\% and $12.3 \%$, respectively). This does not coincide with the preoperative symptomatology stated by Dein et al ${ }^{7}$ who stated dyspnea in $88 \%$, neurological symptoms in $22 \%$, and then $11 \%$ with constitutional manifestations. From our point of view, this lack of similarity returns to the strict and detailed preoperative work-up, and the experienced detailed intraoperative echocardiography, which is particularly helpful and specific. ${ }^{8}$ This enabled us to accidentally discover many cases and upgraded this domain as the fourth commonest symptom. We did not focus on pathology-related symptomatology in our study; however, it is already thoroughly discussed in the literature that tumors of malignant origin usually present more aggressively than benign ones. $^{6}$

Choice of the operative technique and incision is mainly based on tumor localization, morphology, expected pathology, and surgical experience. We tended more toward minimally invasive approach whenever possible. In our experience, after the advent of transesophageal echocardiography, we believe visual inspection of all four cardiac chambers is not necessary. Although Jones et al recommended a sternotomy biatrial approach to allow minimal manipulation of the tumor, provided adequate exposure for complete resection, allow inspection of all four heart chambers, and minimize recurrence. ${ }^{9}$ We were able to reach the four goals of Jones et al throughout using a minimally invasive approach. Most of these minimally invasive patients were mainly addressed to right or left atriotomies, but unfortunately, we recorded conversion to sternotomy between two patients $(0.7 \%)$ because of surgical surprise in the sense of highly invading inaccessible tumors which were not accurately seen through the preoperative imaging tools. Of note, those conversion patients had pathologically papillary fibroelastoma tumor. Some authors also reported low recurrence and safety of these incisions (right or left atriotomy) as well. ${ }^{4,5}$

There is still considerable controversy concerning the extent of resection, usage of patch repairs, or endocardial tumor base ablation. Our policy was to excise until the base but preserving the endomyocardial and intramyocardial thickness then substituting the extended base resection with endocardial ablation. Our results were satisfactory in this domain, recording lower bypass times (mean 94.01 minutes), ischemic times (mean 42.5 minutes), and lower recurrence in five patients (1.85\%). On the contrary, other satisfactory results were reached through only wide excision and patch repair ${ }^{10}$ and some others through only simple excision recording recurrence-free periods of 4.5 to 10 years. $^{11,12}$ In our study, septal defects were repaired usually with bovine pericardial patch. Only one of the five patients needed a septal patch repair. In two patients, a primary repair was sufficient.

Recurrence could be related to various factors such as the primary pathology and extent of resection. ${ }^{13}$ However, it is advised for rapidly growing tumors to be excised with a wide safety margin, and for benign local tumors such as myxomas, it is not always necessary to completely resect the septum or atrial wall. Our recurrence results are better than some authors who found out 5\% recurrence of myxomas $^{14}$ after extended resection. In comparison, there was $1.85 \%$ recurrence in our study. We argue this fact for the addition of endocardial ablation in the base of the tumors as routine step after excision. Moreover, this adds to the theory that minimally invasive approaches are not inferior to sternotomy concerning recurrence rates, since 153 patients (56.8\%) from our study were operated on using this technique.

Our survival rates were studied in correlation to all variables to try to get independent factors of survival. Concerning gender-related survival, we found there were more female survivals than males in the first 7 follow-up years. Beginning from the seventh year, the male survivals were more than females. This could be explained through the fact that our age-gender preoperative correlation showed a highly advanced age in the female group to the male group. We concluded statistically significant higher survival among patients, who were operated upon at age younger than 63 years. Despite this finding, we cannot define an age limit above which surgery is contraindicated because surgical excision remains the main curative modality irrespective of age. Only in special cases do we have to weigh operability, survival, and life quality with age.

Pathology-related survival showed relative equal survival between myxomas and fibroelastomas in the first 7 years of follow-up. After that period, myxoma patients showed statistically significant higher survival log rank $(p<0.01)$ (-Fig. 5).

Our results showed significantly lower survivals among sarcomas and lymphomas. This coincides with Marvasti et $\mathrm{al}^{15}$ concerning myxomas survivals and Poole et al concerning malignancy survivals. ${ }^{16}$ Our explanation of this fact was that

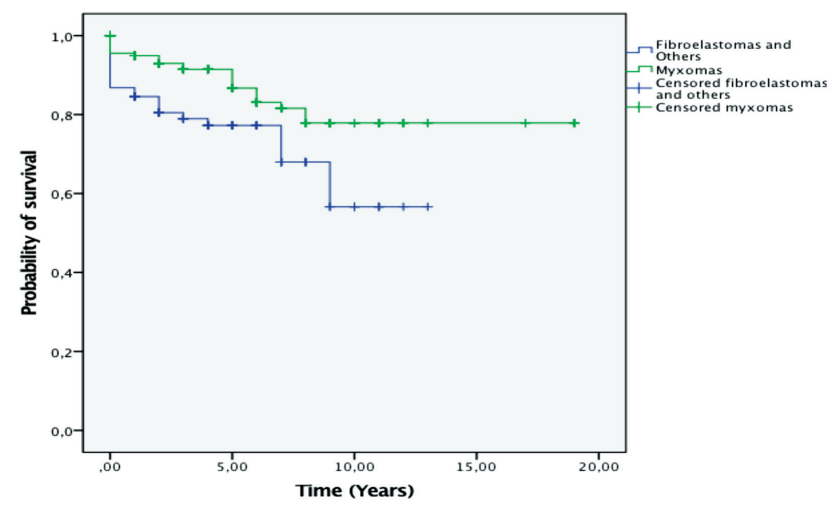

Fig. 5 Kaplan-Meier's survival analysis: myxomas versus others. 
the relative ease of technical feasibility of tumor resection had a significant effect on mean patient survival following surgery. Syncope, as a preoperative symptom had no statistical significance on survival with a log rank $(p=0.89$ ). This could be explained through being the fourth commonest presentation among our study population. That is why we could not record any statistical significance in this domain. Survival statistically significant results concerning the NYHA class preoperatively were detected among our study population scoring a log rank of 0.00 . Patients with NYHA IV preoperatively showed least survivals at 12 postoperative years of follow-up, while patients with no dyspnea as a presenting symptom recorded two plateau phases of stable survival rate, from years 1 to 7 and from year 10 until the end of follow-up. This fact was not studied in detail throughout the published literature in this domain as a result we could not find any comparative results concerning the NYHA-survival relation. The influence of preoperative ejection fraction on survival was also one of our focus points. Our results state statistically insignificant values of $\log$ rank 0.149 . Localization of the cardiac tumor and postoperative arrhythmias was thoroughly studied and recorded statistically significant results with log rank 0.01 in both variables. We have relatively lower incidence of postoperative arrhythmias when compared with other publications. ${ }^{17}$ This may be because of the tumor base cryoablation that we applied as routine step after tumor excision. Being a statistically significant parameter, we see that it is worth to further intensively investigate this as a separate parameter, although throughout our study it had no influence on survival. Finally, bleeding as a postoperative parameter was neither statistically significant nor of influence on survival.

Surgical resection, when possible, is the treatment of choice for all patients with cardiac neoplasms. The results of surgical treatment and survival are closely related and dependent on pathology, age, NYHA preoperatively, tumor localization, and postoperative arrhythmias of the tumors. Specifically, age, pathology, and NYHA preoperatively are dependent factors of survival. A close postoperative longterm follow-up and observation of these patients are recommended. It is curative in benign tumors and may prolong life in malignant tumors, although we think that development of minimally invasive techniques adds for improvement of life quality.

\section{Source of Funding}

None.

\section{Conflict of Interest}

The authors have no conflict of interest to declare.

\section{References}

1 McAllister HA Jr, Fenoglio JJ Jr. Tumors of the cardiovascular system. In: Atlas of tumor pathology. Washington D.C.: Armed forces institute of pathology; 1978

2 Crafoord C. Discussion on mitral stenosis and mitral insufficiency, e. Lam CR, Editor. Philadelphia: W.B. Saunders: Proceedings of the International Symposium on Cardiovascular Surgery Henry Ford Hospital, Detroit. p. 1955.202-11.11

3 Habertheuer A, Laufer G, Wiedemann D, et al. Primary cardiac tumors on the verge of oblivion: a European experience over 15 years. J Cardiothorac Surg 2015;10:56

4 Meyns B, Vancleemput J, Flameng W, Daenen W. Surgery for cardiac myxoma. A 20-year experience with long-term follow-up. Eur J Cardiothorac Surg 1993;7(08):437-440

5 Actis Dato GM, De Benedictis M, Actis Dato A Jr, Ricci A, Sommariva L, De Paulis R. Long-term follow-up of cardiac myxomas (7-31 years). J Cardiovasc Surg (Torino) 1993;34(02):141-143

6 Habertheuer A, Ehrlich M, Wiedemann D, Mora B, Rath C, Kocher A. A rare case of primary cardiac B cell lymphoma. J Cardiothorac Surg 2014;9:14

7 Dein JR, Frist WH, Stinson EB, et al. Primary cardiac neoplasms. Early and late results of surgical treatment in 42 patients. J Thorac Cardiovasc Surg 1987;93(04):502-511

8 Obeid AI, Marvasti M, Parker F, Rosenberg J. Comparison of transthoracic and transesophageal echocardiography in diagnosis of left atrial myxoma. Am J Cardiol 1989;63(13):1006-1008

9 Jones DR, Warden HE, Murray GF, et al. Biatrial approach to cardiac myxomas: a 30-year clinical experience. Ann Thorac Surg 1995;59(04):851-855, discussion 855-856

10 Gerbode F, Kerth WJ, Hill JD. Surgical management of tumors of the heart. Surgery 1967;61(01):94-101

11 Attar S, Lee YC, Singleton R, Scherlis L, David R, McLaughlin JS. Cardiac myxoma. Ann Thorac Surg 1980;29(05):397-405

12 Melo J, Ahmad A, Chapman R, Wood J, Starr A. Primary tumors of the heart: a rewarding challenge. Am Surg 1979;45(11):681-683

13 Sellke FW, Lemmer JH Jr, Vandenberg BF, Ehrenhaft JL. Surgical treatment of cardiac myxomas: long-term results. Ann Thorac Surg 1990;50(04):557-561

14 Kabbani SS, Jokhadar M, Meada R, et al. Atrial myxoma: report of 24 operations using the biatrial approach. Ann Thorac Surg 1994; 58(02):483-487, discussion 487-488

15 Marvasti MA, Obeid AI, Potts JL, Parker FB. Approach in the management of atrial myxoma with long-term follow-up. Ann Thorac Surg 1984;38(01):53-58

16 Poole GV Jr, Meredith JW, Breyer RH, Mills SA. Surgical implications in malignant cardiac disease. Ann Thorac Surg 1983;36(04): 484-491

17 Elbardissi AW, Dearani JA, Daly RC, et al. Survival after resection of primary cardiac tumors: a 48-year experience. Circulation 2008; 118(14, Suppl):S7-S15 